\title{
Analysis of a hybrid zone in Fundulus majalis in a northeastern Florida ecotone
}

\author{
CHARLES F. DUGGINS JR*, ALVAN A. KARLIN†, TIMOTHY A. MOUSSEAU \& KENNETH \\ G. RELYEA $\ddagger$ \\ Department of Biological Sciences, University of South Carolina, Columbia, SC 29208, †Department of Biology, \\ University of Arkansas at Little Rock, Little Rock, AR 72204 and $\ddagger$ Department of Biology, Armstrong State College, \\ Savannah, GA 31419, U.S.A.
}

\begin{abstract}
Fundulus majalis and $F$. similis are small fish that inhabit the coastal waters of eastern North America. Fundulus majalis occurs from New Hampshire to northeastern Florida where it is replaced by $F$. similis. Past morphological studies of these species have suggested the existence of a hybrid zone in the Flagler County area of northeast Florida; this location also corresponds to an ecological transition from northern Juncus-Spartina marsh to southern mangrove marsh. We used protein electrophoresis to test the hypothesis that there is a significant barrier to gene flow between the two species in the area of Flagler County, FL. Twenty populations were surveyed electrophoretically for 26 protein producing loci. Significant allozyme variation was observed for five loci: adenosine deaminase, an esterase, isocitrate dehydrogenase, malate dehydrogenase and phosphoglucomutase. Allele frequencies at these loci vary clinally from a 'majalis'-like distribution in more northern localities to a 'similis'-like distribution in southern localities. The steepest portion of the cline occurs over a $30 \mathrm{~km}$ portion of intracoastal salt marsh bordered by the northern and southern boundaries of Flagler County, FL, which led us to examine eight populations from within this area. We speculate that the nominal $F$. majalis and $F$. similis were allopatric and in the process of speciation when they recontacted one another, and the process of differentiation broke down and secondary intergradation occurred. The width of the genotypic clines (only $s M d h-A$ is fixed at both ends), $\mathrm{Nm}$ estimates, and the absence of significant linkage disequilibria support the hypothesis that the rapid shift in genotypes and morphology in Flagler County is a dispersal-independent cline maintained by selection across the rapid ecological transition of an ecotone.
\end{abstract}

Keywords: cline, Cyprinodontidae, ecotone, Fundulus, hybrid zone, linkage disequilibria.

\section{Introduction}

Hybrid zones are regions where genetically different populations meet and interbreed to produce hybrids (Barton \& Hewitt, 1989). Hybrid zones appear to be common; Hewitt (1989) noted that in many groups one-third to one-half of the species are subdivided by hybrid zones. Hybrid zones may be wide or narrow, and may or may not be associated with ecotones.

Hybrid zones fall into three principal categories: those maintained by selection against hybrids (tension zones), those maintained by selection along an environmental gradient and those in which there is equivalent selection on the allelic differences between the two races resulting in the cline of allele frequencies in the

*Correspondence. hybrid zone becoming more and more shallow with time (Hewitt, 1989).

In cases in which species occur in abutting habitats, and if they hybridize where they come into contact, the resulting hybrid zone will be in the ecotone produced where the habitats abut. Hewitt (1989) observed that many hybrid zones occur along ecotones. In these cases one may observe parallel clines in allele frequencies across many genetic loci. These clines in allele frequencies seemingly closely parallel clines in environmental parameters (e.g. temperature, salinity). Other loci may not vary clinally; presumably these loci are not affected by selection by the parameters that vary across the ecotone.

Alternatively, a rapid environmental gradient may lead to a hybrid zone within a species. This could result from strong selection across many loci in response to 
selection along an environmental gradient across a short geographical distance. Brown \& Chapman (1991) demonstrated a situation in two subspecies of Fundulus heteroclitus where two populations which had apparently diverged in allopatry maintained some of their differences after recontact with a cline in characters across an environmental gradient.

The question is, therefore, how to differentiate strong clines in morphology and allelic isozymes which result from hybridization and restricted gene flow across an ecotone vs. those clines which result entirely from selection on the populations of a species which happen to cross an ecotone, i.e. the presence of a hybrid zone even though there is no barrier to gene flow.

The Fundulus majalis-Fundulus similis species complex includes three nominal species: $F$. majalis (Walbaum), F. similis (Girard) and $F$. persimilis Miller. They are small fishes of the family Cyprinodontidae and typically occur in brackish waters of intracoastal waterways, low energy coastlines and estuarine lagoons. Fundulus majalis ranges continuously, or nearly so, along the coast from New Hampshire (Jackson, 1953; Brown, 1957) southward to northeastern Florida. Except for the region in southeast Florida between Lake Worth and Miami, Fundulus similis is continuously distributed from northeastern Florida around the Florida peninsula and along the coast of the Gulf of Mexico to Tampico, Mexico (Miller, 1955; Relyea, 1983).

Based on presumed sympatry of the two forms in the Matanzas River in northeastern Florida, approximately $25 \mathrm{~km}$ north of the Flagler Beach area, Miller (1955) concluded that $F$. majalis and $F$. similis were distinct species. Brown (1957) also recognized both species, and in his key to the species of Fundulus used head length to distinguish them. However, Relyea (1983) concluded that $F$. majalis and $F$. similis were conspecific. He showed that from north to south along the Atlantic coast head length, pigmentary patterns and other diagnostic characters varied continuously from $F$. majalis-like to $F$. similis-like. Relyea (1983) also suggested that gene flow from the nominal $F$. majalis into the disjunct Florida east coast population of the nominal $F$. similis had distinguished this population from other $F$. similis populations in the Florida Keys and Gulf of Mexico.

When Relyea (1983) concluded that $F$. majalis and $F$. similis were conspecific, he reported that the diagnostic features changed sharply as geographical samples were measured along a north-south transect over a linear distance of only $30 \mathrm{~km}$ in a coastal marsh in northeastern Florida. The purpose of this investigation was to determine the extent and nature of gene flow between the two nominal forms in this $30 \mathrm{~km}$ of marsh, and therefore to test Relyea's (1983) hypothesis that they are conspecific. The results should also provide insight into the nature of hybrid zones.

\section{Materials and methods}

\section{Materials}

Specimens were collected from the 20 localities listed below, placed on dry ice and returned to the laboratory where they were stored at $-70^{\circ} \mathrm{C}$ until used for electrophoresis. The localities were chosen based on morphological and meristic evidence described in an earlier study of the species complex (Relyea, 1983). Sixty individuals per locality were assayed except for the Georgia locality (sample 2, 38 individuals) and the Carrabelle, Florida locality (sample 20, 20 individuals). The sample numbers correspond to those of Fig. 1 and in the tables.

The geographical samples were collected at North Carolina: (1) Brunswick Co., Wrightsville Beach; Georgia: (2) Chatham Co., Oaussau Island; Florida: (3) St. Johns Co., Matanzas Inlet; (4) Flagler Co., 13 km S. of the Flagler Co. line; (5) Flagler Co., $14.5 \mathrm{~km}$ S. of the Flagler Co. line; (6) Flagler Co., $16 \mathrm{~km} \mathrm{~S}$. of the Flagler Co. line; (7) Flagler Co., $17.5 \mathrm{~km} \mathrm{~S}$. of the Flagler Co. line; (8) Flagler Co., $18.5 \mathrm{~km}$ S. of the Flagler Co. line; (9) Flagler Co., $19.3 \mathrm{~km} \mathrm{~S}$. of the Flagler Co. line; (10) Flagler Co., $20 \mathrm{~km} \mathrm{~S}$. of the Flagler Co. line; (11) Flagler Co., Rte 100 at intracoastal waterway, 22.25 $\mathrm{km} \mathrm{S}$. of the Flagler Co. line; (12) Flagler Co., $0.8 \mathrm{~km} \mathrm{~N}$ of Flagler Beach State Park, 26km S. of the Flagler Co. line; (13) Brevard Co., Rte 40 at the intracoastal waterway; (14) Brevard Co., SR 404 at the intracoastal waterway near Satellite Beach; (15) Monroe Co., Grassy Key; (16) Monroe Co., Big Pine Key; (17) Collier Co., Marco Island; (18) Pinellas Co., Ft. DeSoto Co. Park; (19) Dixie Co., Horseshoe Beach; (20) Franklin Co., Carrabelle.

\section{Electrophoretic methods}

Protein samples for electrophoresis were obtained by homogenizing individual fish in an equal volume of chilled distilled water. The resultant slurry was centrifuged at $25000 \mathrm{~g}$ at $4^{\circ} \mathrm{C}$ for $60 \mathrm{~min}$. The supernatant of water soluble proteins was decanted and stored at $4^{\circ} \mathrm{C}$ overnight, a maximum of $18 \mathrm{~h}$ prior to electrophoretic separations. To determine the specific origin of each enzyme studied, organ extracts were analysed separately.

In a preliminary survey, 26 proteins were routinely surveyed in 20 individuals from each population (Table 
Fig. 1 Collection localities and population numbers of Fundulus samples. Populations 4-12 are located from north to south in numerical order and are each approximately $1-4 \mathrm{~km}$ apart. See Materials and methods for precise localities.

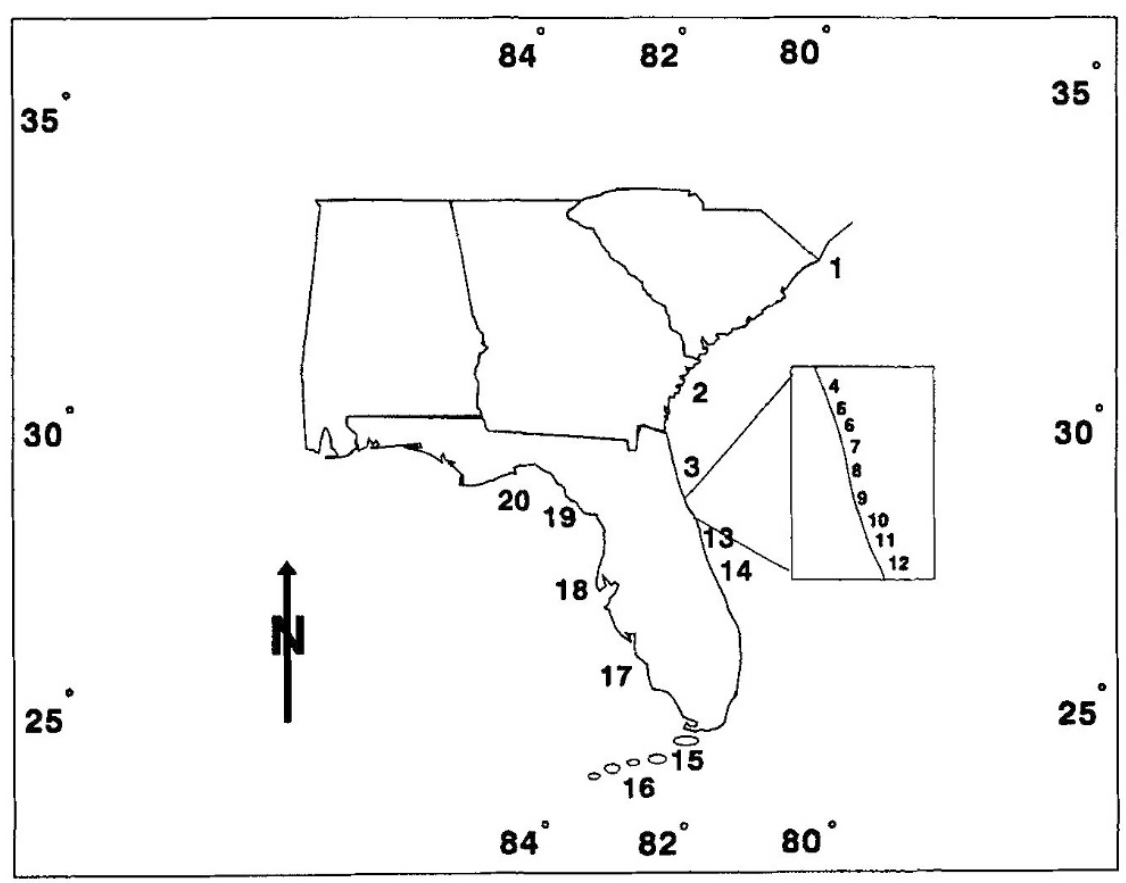

1). Following the initial survey, five polymorphic systems ( $A d a-A$, Est-3, sIcdh- $A$, sMdh- $A$, and Pgm$A$ ) were evaluated for 40 additional individuals of most populations. However, as our survey proceeded and the pattern of genotypic variation became evident, we surveyed additional loci in an attempt to gain more information. This survey consisted of comparing five fish from a 'majalis' locality (population 2, near Savannah, Georgia) to five fish from a 'similis' locality (population 20, near Carrabelle, Florida). These loci were acid phosphatase, adenylate kinase, alcohol dehydrogenase, aldehyde oxidase, aldolase, creatine kinase, fumarase, beta galactosidase, glutamate dehydrogenase, glycerol dehydrogenase, hexokinase, mannose phosphate isomerase, nucleoside phosphorylase, octanol dehydrogenase, sorbitol dehydrogenase, succinate dehydrogenase and xanthine dehydrogenase. No important variation was found at these loci, and they were no longer assayed.

Our techniques of starch gel electrophoresis were similar to those described by Brewer (1970) and Selander et al. (1971) (see Table 1 for electrophoretic conditions). The stain for Gludh was modified from Buth \& Berg (1979) to contain $9.0 \mathrm{~g}$ D-glucose, $0.005 \mathrm{~g}$ each of NBT, MTT, PMS and $0.01 \mathrm{~g}$ of NADP. All gels were 13 per cent starch (Electrostarch lots 392, 307 and 84 , Otto Hiller Electrostarch Company, Madison, Wisconsin). Isozyme separation was essentially the same on different lots of Electrostarch, although lot 84 required approximately 20 per cent longer running times. The locus nomenclature follows Fisher et al.
(1980) and Crabtree \& Buth (1981). When electromorph (allelic) variation was observed, the electromorph with the greatest anodal migration was called $a$, the next $b$, and so on.

\section{Analytical methods}

Individual genotypes for each protein were assigned based on standard methods (Brewer, 1970) of gel interpretation. These presumed genotypes were entered into BIOSYS-1 (Swofford and Selander, 1981) for computer analysis.

To determine general patterns of gene frequency distributions and genetic differentiation among populations, Nei's (1978) genetic identity and distance estimates were calculated. These methods were used to construct phenograms by the UPGMA method. Additional clustering algorithms were used and the method that yielded the greatest cophenetic correlation was chosen.

As another measure of genetic differentiation we computed Wright's (1978) F-statistics. In one population model that we used, the total population $(T)$ was subdivided into two subpopulations $(\mathbf{S})$ on the basis of species and the geographical samples $(I)$ were considered as individual units of their respective species. Because Relyea (1983) suggested that differentiation may exist within local clusters of populations of $F$. similis, we analysed another population model. In this model, four subpopulations ( $\mathrm{S}$ ) of the total ( $\mathrm{T}$ ) population were defined based on a Nei's genetic identity of 
Table 1 Enzymes, loci and electrophoretic conditions

\begin{tabular}{|c|c|c|}
\hline Enzyme (E.C. number) & Locus & $\begin{array}{c}\text { Electrophoretic } \\
\text { conditions } \dagger\end{array}$ \\
\hline $\begin{array}{l}\text { Adenosine deaminase } \\
\quad \text { (E.C. } 3.5 .4 .4)\end{array}$ & $A d a-A$ & TC 8.0 \\
\hline \multirow{2}{*}{$\begin{array}{l}\text { Aspartate aminotransferase } \\
\text { (E.C. 2.6.1.1) }\end{array}$} & $M-A a t-A$ & TC 8.0 \\
\hline & $S-A a t-A$ & TC 8.0 \\
\hline Catalase (E.C. 1.11.1.6) & Cat & TC 8.0 \\
\hline \multirow[t]{3}{*}{ Esterase (nonspecific) } & Est-1 & $\mathrm{LiOH}$ \\
\hline & Est-2 & $\mathrm{LiOH}$ \\
\hline & Est-3 & $\mathrm{LiOH}$ \\
\hline $\begin{array}{l}\text { Glucose dehydrogenase } \\
\text { (E.C. } 1.1 .1 .47)\end{array}$ & Gludh & TM 7.4 \\
\hline \multirow{2}{*}{$\begin{array}{l}\text { Glucose phosphate isomerase } \\
\text { (E.C. 5.3.1.9) }\end{array}$} & $G p i-A$ & $\mathrm{LiOH}$ \\
\hline & $G p i-B$ & $\mathrm{LiOH}$ \\
\hline $\begin{array}{l}\text { Glutamate dehydrogenase } \\
\text { (E.C. 1.4.1.3) }\end{array}$ & $G d h$ & TM 7.4 \\
\hline $\begin{array}{l}\text { Glyceraldehyde-phosphate } \\
\text { dehydrogenase (E.C. 1.2.1.9) }\end{array}$ & Gapdh-A & TM 7.4 \\
\hline \multirow{2}{*}{$\begin{array}{l}\text { Isocitrate dehydrogenase } \\
\text { (E.C. 1.1.1.42) }\end{array}$} & $m I c d h-A$ & TC 8.0 \\
\hline & sIcdh-A & TC 8.0 \\
\hline \multirow{3}{*}{$\begin{array}{l}\text { Lactate dehydrogenase } \\
\quad \text { (E.C. } 1.1 .1 .27)\end{array}$} & $L d h-A$ & TM 7.4 \\
\hline & $L d h-B$ & TM 7.4 \\
\hline & $L d h-C$ & TM 7.4 \\
\hline \multirow{2}{*}{$\begin{array}{l}\text { Malate dehydrogenase } \\
\text { (E.C. } 1.1 .1 .37)\end{array}$} & $s M d h-A$ & TC 8.0 \\
\hline & $s M d h-B$ & TC 8.0 \\
\hline \multirow{2}{*}{$\begin{array}{l}\text { Malate dehydrogenase } \\
\quad \text { (E.C. } 1.1 .1 .40)\end{array}$} & $m M e-A$ & TM 7.4 \\
\hline & $s M e-B$ & TM 7.4 \\
\hline \multirow{2}{*}{$\begin{array}{l}\text { Phosphoglucomutase } \\
\text { (E.C. 2.7.5.1) }\end{array}$} & $P g m-A$ & TC 8.0 \\
\hline & $P g m-B$ & TC 8.0 \\
\hline $\begin{array}{l}\text { Phosphogluconate } \\
\text { dehydrogenase (EC 1.1.1.44) }\end{array}$ & Pgdh-A & TC 8.0 \\
\hline Superoxide dismutase & sSod-A & TM 7.4 \\
\hline
\end{tabular}

$\nmid \mathrm{LiOH}$ : lithium hydroxide (Selander et al., 1971), $0.04 \mathrm{~W} / \mathrm{cc}$, $4.5 \mathrm{~h}$; TC 8.0: Tris-citrate pH 8.0 (Selander et al., 1971), 0.03 $\mathrm{W} / \mathrm{cc}, 5 \mathrm{~h}$ (lot nos. 392 and 307), $0.04 \mathrm{~W} / \mathrm{cc}, 5 \mathrm{~h}$ (lot no. 84); TM 7.4: Tris-maleate-EDTA (Selander et al., 1971), 0.037 $\mathrm{W} / \mathrm{cc}, 5 \mathrm{~h}$.

0.98 , as evident on the phenogram (Fig. 2). For both population models, $F_{\mathrm{ST}}$ represents the variance in allele frequencies among subpopulations, $F_{\text {IT }}$ represents the reduction in heterozygosity of an individual relative to the total population, and $F_{\text {Is }}$ represents the reduction in heterozygosity of an individual due to nonrandom mating within its subpopulation.

To determine whether observed allele frequencies and genotypic frequencies were in agreement with Hardy-Weinberg predictions, we first tested each geographical sample for each locus individually. Following this analysis, we used $\chi^{2}$ to determine homogeneity within each nominal species and within the entire sample. We used Slatkin's (1985) method to estimate the degree of gene flow through the area of transition (Flagler County) of the two nominal species.

We estimated linkage disequilibria within populations using the maximum likelihood methods of Weir \& Cockerham (1989). Disequilibria were estimated using the FORTRAN listing provided by Weir (1990). This method produces six disequilibrium coefficients from two-locus, two-allele data. Weir \& Cockerham's (1989) model permits the simultaneous estimation of the two single-locus disequilibria, $D_{\mathrm{A}}$ and $D_{\mathrm{B}}$, the composite digenic disequilibrium $\Delta_{A B}$, the two trigenic disequilibrium estimates $D_{\mathrm{ABB}}$ and $D_{\mathrm{AAB}}$, and the composite quadragenic disequilibrium estimate $\Delta_{\mathrm{AABB}}$. Because five loci varied along the cline from $F$. majalis in the north to $F$. similis in the south, we calculated a total of 10 disequilibrium estimates of each type for each population.

\section{Results}

\section{Electrophoretic results}

Of the 27 loci coding for proteins surveyed in this study, 19 were monomorphic or nearly so (frequency of the common allele in all populations >0.95). Although Est-2 and Cat were polymorphic, they were difficult to score consistently and were omitted from analysis. The observed genotypes for the remaining five polymorphic loci are reported in Table 2.

When gene frequencies were used to estimate genetic distance and similarities, Nei's (1978) identity values varied from 0.832 to 0.999 and distance values varied from 0.184 to 0.001 (the full matrix is available from A.A.K. on request). The pattern of identity values is apparent from the phenogram (Fig. 2) which clearly clusters samples. The cluster of samples 1-6 represents the nominal $F$. majalis and the northern one-quarter of the transition zone (Flagler County), 7-10 represent the more central samples from the transition zone whereas samples 11-14 represent the southern onequarter of the transition zone and samples from the allopatric Florida east coast population of the nominal F. similis; 15 and 16 are Florida Keys samples of the nominal $F$. similis and 17-20 are Florida Gulf coast samples. The clustering pattern is consistent with Relyea's (1983) suggestion that Florida east coast samples of the nominal $F$. similis $(7-14)$ can be distinguished from Florida Keys and Florida Gulf coast samples $(15-20)$.

\section{Genetic results}

Of the $100 \chi^{2}$ tests performed (five loci in each of 20 geographical samples), 92 showed no significant 


\section{Cophenetic Correlation $=0.705$}

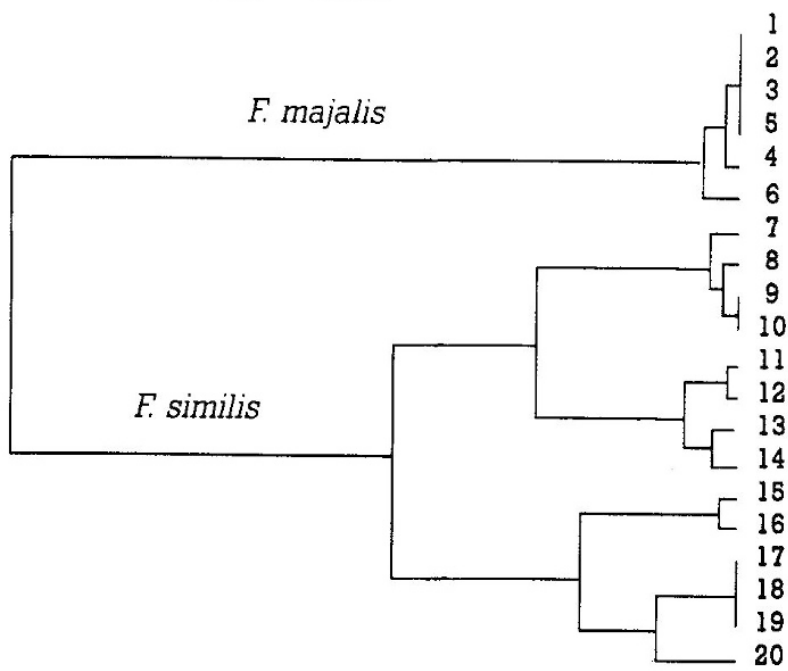

Fig. 2 Phenogram for Fundulus

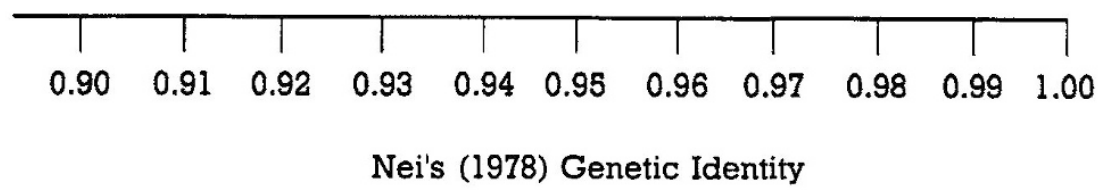
genetic identity values.

Nei's (1978) Genetic Identity

Table 2 Genotypic distributions for five loci for 20 populations of Fundulus

\begin{tabular}{|c|c|c|c|c|c|}
\hline Population & Ada- $A$ & Est-3 & sIcdh-A & $s M d h-A$ & Pgm- $A$ \\
\hline (1) Wrightsville Beach & $\mathrm{bb}(60)$ & $\begin{array}{l}\operatorname{dd}(2) \\
\operatorname{de}(17) \\
\operatorname{df}(1) \\
\text { ee }(38) \\
\text { ef }(2)\end{array}$ & $\begin{array}{l}\mathrm{bb}(38) \\
\mathrm{bc}(18) \\
\mathrm{cc}(4)\end{array}$ & aa $(60)$ & aa $(60)$ \\
\hline (2) Oaussau Island & $\begin{array}{l}\mathrm{ab}(1) \\
\mathrm{bb}(36) \\
\mathrm{bc}(1)\end{array}$ & $\begin{array}{l}\text { ce }(2) \\
\text { dd }(2) \\
\text { de }(11) \\
\text { ee }(23)\end{array}$ & $\begin{array}{l}\mathrm{bb}(18) \\
\mathrm{bc}(17) \\
\mathrm{cc}(3)\end{array}$ & aa $(38)$ & $\begin{array}{l}a+a(2) \\
a+b(1) \\
a a(35)\end{array}$ \\
\hline (3) Matanzas Inlet & $\begin{array}{l}\mathrm{bb}(54) \\
\mathrm{bc}(6)\end{array}$ & $\begin{array}{l}\text { bc }(1) \\
\text { bd }(1) \\
\text { be }(4) \\
\operatorname{cc}(2) \\
\operatorname{ce}(5) \\
\operatorname{dd}(1) \\
\operatorname{de}(9) \\
\text { ee }(37)\end{array}$ & $\begin{array}{l}\mathrm{ab}(1) \\
\mathrm{ac}(1) \\
\mathrm{bb}(24) \\
\mathrm{bc}(28) \\
\mathrm{cc}(6)\end{array}$ & $\begin{array}{l}\mathrm{aa}(58) \\
\mathrm{ab}(2)\end{array}$ & $\begin{array}{l}\mathrm{aa}(58) \\
\mathrm{ab}(2)\end{array}$ \\
\hline (4) $13 \mathrm{~km} \mathrm{~S}$. Flagler Co. line & $\begin{array}{l}\mathrm{bb}(50) \\
\mathrm{bc}(9) \\
\mathrm{cc}(1)\end{array}$ & $\begin{array}{l}\operatorname{cc}(1) \\
\operatorname{ce}(4) \\
\operatorname{de}(10) \\
\operatorname{ee}(45)\end{array}$ & $\begin{array}{l}\mathrm{ab}(1) \\
\mathrm{ac}(1) \\
\mathrm{bb}(20) \\
\mathrm{bc}(27) \\
\mathrm{cc}(11)\end{array}$ & $\begin{array}{l}\mathrm{aa}(57) \\
\mathrm{ab}(3)\end{array}$ & $\begin{array}{l}\mathrm{aa}(58) \\
\mathrm{ab}(2)\end{array}$ \\
\hline (5) $14.5 \mathrm{~km} \mathrm{~S}$. Flagler Co. line & $\begin{array}{l}\mathrm{bb}(49) \\
\mathrm{bc}(11)\end{array}$ & $\begin{array}{l}\text { bc }(2) \\
\text { bd }(1) \\
\text { be }(5) \\
\text { ce }(3) \\
\text { dd }(1) \\
\text { de }(10) \\
\text { ee }(38)\end{array}$ & $\begin{array}{l}\mathrm{ab}(2) \\
\mathrm{bb}(30) \\
\mathrm{bc}(21) \\
\mathrm{cc}(7)\end{array}$ & $\begin{array}{l}\mathrm{aa}(55) \\
\mathrm{ab}(5)\end{array}$ & $\begin{array}{l}\mathrm{aa}(57) \\
\mathrm{ab}(3)\end{array}$ \\
\hline
\end{tabular}


Table 2 Continued

\begin{tabular}{|c|c|c|c|c|c|}
\hline Population & $A d a-A$ & Est-3 & $s I c d h-A$ & $s M d h-A$ & $P g m-A$ \\
\hline (6) $16 \mathrm{~km} \mathrm{S.} \mathrm{Flagler} \mathrm{Co.} \mathrm{line}$ & $\begin{array}{l}\mathrm{bb}(31) \\
\mathrm{bc}(19) \\
\mathrm{cc}(10)\end{array}$ & $\begin{array}{l}\mathrm{bb}(1) \\
\mathrm{bc}(2) \\
\mathrm{be}(6) \\
\mathrm{cc}(1) \\
\mathrm{cd}(4) \\
\mathrm{ce}(14) \\
\mathrm{dd}(1) \\
\operatorname{de}(7) \\
\mathrm{ee}(24)\end{array}$ & $\begin{array}{l}\text { aa }(1) \\
\mathrm{ab}(9) \\
\mathrm{ac}(3) \\
\mathrm{bb}(20) \\
\mathrm{bc}(22) \\
\mathrm{cc}(5)\end{array}$ & $\begin{array}{l}\mathrm{aa}(49) \\
\mathrm{ab}(11)\end{array}$ & $\begin{array}{l}\mathrm{aa}(45) \\
\mathrm{ab}(11) \\
\mathrm{ac}(1) \\
\mathrm{bb}(3)\end{array}$ \\
\hline (7) $17.5 \mathrm{~km}$ S. Flagler Co. line & $\begin{array}{l}\mathrm{bb}(26) \\
\mathrm{bc}(27) \\
\mathrm{cc}(7)\end{array}$ & $\begin{array}{l}\mathrm{bb}(1) \\
\mathrm{bc}(5) \\
\mathrm{bd}(1) \\
\mathrm{be}(5) \\
\mathrm{cc}(7) \\
\mathrm{cd}(3) \\
\operatorname{ce}(17) \\
\operatorname{de}(4) \\
\operatorname{ee}(17)\end{array}$ & $\begin{array}{l}\text { aa }(4) \\
\mathrm{ab}(23) \\
\mathrm{ac}(4) \\
\mathrm{bb}(16) \\
\mathrm{bc}(10) \\
\mathrm{cc}(3)\end{array}$ & $\begin{array}{l}a+a(1) \\
a a(26) \\
a b(23) \\
b b(10)\end{array}$ & $\begin{array}{l}\mathrm{aa}(40) \\
\mathrm{ab}(18) \\
\mathrm{bb}(2)\end{array}$ \\
\hline (8) $18.5 \mathrm{~km} \mathrm{~S}$. Flagler Co. line & $\begin{array}{l}\mathrm{bb}(14) \\
\mathrm{bc}(29) \\
\mathrm{cc}(16)\end{array}$ & $\begin{array}{l}\mathrm{bb}(1) \\
\mathrm{bc}(7) \\
\mathrm{bd}(1) \\
\mathrm{be}(3) \\
\mathrm{cc}(8) \\
\mathrm{cd}(4) \\
\mathrm{ce}(19) \\
\mathrm{dd}(1) \\
\mathrm{de}(4) \\
\mathrm{ee}(11)\end{array}$ & $\begin{array}{l}\mathrm{aa}(9) \\
\mathrm{ab}(20) \\
\mathrm{ac}(5) \\
\mathrm{bb}(7) \\
\mathrm{bc}(14) \\
\mathrm{cc}(4)\end{array}$ & $\begin{array}{l}\mathrm{aa}(23) \\
\mathrm{ab}(20) \\
\mathrm{bb}(16)\end{array}$ & $\begin{array}{l}\mathrm{aa}(41) \\
\mathrm{ab}(13) \\
\mathrm{bb}(4) \\
\mathrm{bc}(1)\end{array}$ \\
\hline (9) $19.3 \mathrm{~km}$ S. Flagler Co. line & $\begin{array}{l}\mathrm{bb}(18) \\
\mathrm{bc}(32) \\
\mathrm{cc}(10)\end{array}$ & $\begin{array}{l}\mathrm{ac}(1) \\
\mathrm{bb}(1) \\
\mathrm{bc}(4) \\
\mathrm{bd}(2) \\
\mathrm{be}(3) \\
\mathrm{cc}(15) \\
\mathrm{cd}(2) \\
\mathrm{ce}(25) \\
\mathrm{cf}(1) \\
\operatorname{de}(2) \\
\operatorname{ee}(4)\end{array}$ & $\begin{array}{l}\mathrm{aa}(8) \\
\mathrm{ab}(26) \\
\mathrm{ac}(9) \\
\mathrm{bb}(14) \\
\mathrm{bc}(2) \\
\mathrm{cc}(1)\end{array}$ & $\begin{array}{l}\mathrm{aa}(17) \\
\mathrm{ab}(28) \\
\mathrm{bb}(15)\end{array}$ & $\begin{array}{l}\mathrm{aa}(31) \\
\mathrm{ab}(24) \\
\mathrm{bb}(5)\end{array}$ \\
\hline (10) $20 \mathrm{~km}$ S. Flagler Co. line & $\begin{array}{l}\mathrm{bb}(20) \\
\mathrm{bc}(25) \\
\mathrm{cc}(15)\end{array}$ & $\begin{array}{l}\text { bc }(11) \\
\text { be }(9) \\
\operatorname{cc}(12) \\
c d(1) \\
\operatorname{ce}(18) \\
\operatorname{de}(2) \\
\operatorname{ee}(7)\end{array}$ & $\begin{array}{l}\mathrm{aa}(12) \\
\mathrm{ab}(16) \\
\mathrm{ac}(10) \\
\mathrm{bb}(17) \\
\mathrm{bc}(5)\end{array}$ & $\begin{array}{l}\mathrm{aa}(15) \\
\mathrm{ab}(27) \\
\mathrm{bb}(18)\end{array}$ & $\begin{array}{l}\mathrm{aa}(34) \\
\mathrm{ab}(21) \\
\mathrm{bb}(3)\end{array}$ \\
\hline (11) $22.25 \mathrm{~km} \mathrm{~S}$. Flagler Co. line & $\begin{array}{l}\mathrm{bb}(12) \\
\mathrm{bc}(31) \\
\mathrm{cc}(17)\end{array}$ & $\begin{array}{l}\mathrm{bb}(1) \\
\mathrm{bc}(12) \\
\mathrm{be}(3) \\
\mathrm{cc}(23) \\
\mathrm{cd}(2) \\
\mathrm{ce}(15) \\
\operatorname{de}(1) \\
\mathrm{ee}(3)\end{array}$ & $\begin{array}{l}\mathrm{aa}(12) \\
\mathrm{ab}(25) \\
\mathrm{ac}(2) \\
\mathrm{bb}(15) \\
\mathrm{bc}(6)\end{array}$ & $\begin{array}{l}\mathrm{aa}(3) \\
\mathrm{ab}(27) \\
\mathrm{bb}(30)\end{array}$ & $\begin{array}{l}\mathrm{aa}(34) \\
\mathrm{ab}(20) \\
\mathrm{ac}(1) \\
\mathrm{bb}(4) \\
\mathrm{bc}(1)\end{array}$ \\
\hline (12) 26 km S. Flagler Co. line & $\begin{array}{l}\mathrm{bb}(9) \\
\mathrm{bc}(26) \\
\mathrm{cc}(25)\end{array}$ & $\begin{array}{l}\mathrm{bb}(3) \\
\mathrm{bc}(1) \\
\mathrm{bd}(1) \\
\mathrm{be}(3) \\
\mathrm{cc}(22) \\
\mathrm{cd}(4) \\
\operatorname{ce}(20) \\
\operatorname{de}(1) \\
\operatorname{ee}(5)\end{array}$ & $\begin{array}{l}\text { aa }(11) \\
\text { ab }(37) \\
\text { ac }(3) \\
\text { bb (7) } \\
\text { bc }(2)\end{array}$ & $\begin{array}{l}\mathrm{aa}(2) \\
\mathrm{ab}(26) \\
\mathrm{bb}(32)\end{array}$ & $\begin{array}{l}\mathrm{aa}(24) \\
\mathrm{ab}(30) \\
\mathrm{bb}(6)\end{array}$ \\
\hline
\end{tabular}


Table 2 Continued

\begin{tabular}{|c|c|c|c|c|c|}
\hline Population & $A d a-A$ & Est-3 & sIcdh-A & $s M d h-A$ & $P g m-A$ \\
\hline (13) Rte 40 & $\begin{array}{l}\mathrm{bb}(1) \\
\mathrm{bc}(18) \\
\mathrm{cc}(41)\end{array}$ & $\begin{array}{l}\mathrm{ab}(1) \\
\mathrm{bb}(5) \\
\mathrm{bc}(18) \\
\mathrm{be}(2) \\
\mathrm{cc}(32) \\
\mathrm{cd}(1) \\
\mathrm{ce}(1)\end{array}$ & $\begin{array}{l}\mathrm{aa}(6) \\
\mathrm{ab}(22) \\
\mathrm{ac}(5) \\
\mathrm{bb}(15) \\
\mathrm{bc}(11) \\
\mathrm{cc}(1)\end{array}$ & $\begin{array}{l}\mathrm{aa}(1) \\
\mathrm{ab}(10) \\
\mathrm{bb}(49)\end{array}$ & $\begin{array}{l}\mathrm{aa}(35) \\
\mathrm{ab}(20) \\
\mathrm{bb}(5)\end{array}$ \\
\hline (15) Grassy Key & $\begin{array}{l}\operatorname{bc}(8) \\
\operatorname{cc}(51) \\
\operatorname{cd}(1)\end{array}$ & $\begin{array}{l}\mathrm{aa}(5) \\
\mathrm{ab}(1) \\
\mathrm{ac}(7) \\
\mathrm{bb}(4) \\
\mathrm{bc}(22) \\
\mathrm{cc}(21)\end{array}$ & $\mathrm{aa}(60)$ & $\mathrm{bb}(60)$ & $\begin{array}{l}\text { aa }(42) \\
\mathrm{ab}(13) \\
\mathrm{bb}(5)\end{array}$ \\
\hline (16) Big Pine Key & $\begin{array}{l}\text { bc }(8) \\
\operatorname{cc}(45) \\
\text { cd }(4) \\
\text { ce }(2) \\
\text { dd }(1)\end{array}$ & $\begin{array}{l}\mathrm{aa}(13) \\
\mathrm{ab}(9) \\
\mathrm{ac}(3) \\
\mathrm{bb}(12) \\
\mathrm{bc}(14) \\
\mathrm{bd}(2) \\
\mathrm{cc}(7)\end{array}$ & $\mathrm{aa}(60)$ & $\mathrm{bb}(60)$ & $\begin{array}{l}\mathrm{aa}(27) \\
\mathrm{ab}(23) \\
\mathrm{bb}(10)\end{array}$ \\
\hline (17) Marco Island & $\begin{array}{l}\mathrm{bb}(2) \\
\mathrm{bc}(12) \\
\mathrm{cc}(46)\end{array}$ & $\begin{array}{l}\text { bb (1) } \\
\text { bc }(17) \\
\mathrm{cc}(31) \\
\mathrm{cd}(11)\end{array}$ & aa $(60)$ & $\mathrm{bb}(60)$ & $\begin{array}{l}\mathrm{aa}(4) \\
\mathrm{ab}(26) \\
\mathrm{bb}(30)\end{array}$ \\
\hline (20) Carrabelle & $\begin{array}{l}\mathrm{bc}(1) \\
\operatorname{cc}(19)\end{array}$ & $\begin{array}{l}\mathrm{ac}(1) \\
\mathrm{bb}(9) \\
\mathrm{bc}(9) \\
\mathrm{cc}(1)\end{array}$ & aa $(20)$ & $\mathrm{bb}(20)$ & $\begin{array}{l}\mathrm{aa}(3) \\
\mathrm{ab}(6) \\
\mathrm{bb}(11)\end{array}$ \\
\hline
\end{tabular}

The number of individuals of each genotype is in parentheses.

departure in genotype distribution from the Hardy-Weinberg model $(P>0.05$; adjusted for the number of tests performed; Harris, 1985). Of the remaining eight cases, which is close to what one would expect by chance alone, there was one (sample 14: Est3 ) in which an unexpected homozygote (ee) was observed whereas the other seven cases each involved a homozygote excess (sample 6: $A d a-A$; sample 8: $S$ -
Mdh-B and Pgm-A ; sample 12: Est-3; sample 15: Est-3 and Pgm-A; sample 16: Est-3). As in most of these cases the departure could be explained by one or a few individuals, we suggest that no systematic error in scoring affected our results.

Wahlund's Effect (Wahlund, 1928) can result when a population consists of a group of heterogeneous subsamples. If there is heterogeneity in allele frequencies 
among subsamples, there will be a net deficiency of heterozygotes and an excess of homozygotes even if Hardy-Weinberg proportions are maintained within each subsample. When we pooled all samples and used BIOSYS-1 to compute $\chi^{2}$ of homogeneity considering each sample as a population, we found statistical significance $(P \ll 0.05)$ for each locus. Similar results were obtained when we used groups of populations as defined by the dendrogram (samples 1-6, 7-20) for groups of samples representing each nominal species. These results suggest that allele frequency heterogeneity exists between samples of the nominal species.

As another measure of population differentiation, hierarchial $F$-statistics (Nei, 1978) were calculated for two slightly different single-level hierarchial models. In one model, we considered samples of each nominal species to represent colonies of a subpopulation of the total. In this case, samples 1-6 represented $F$. majalis and samples 7-20 represented $F$. similis. For this model 25.3 per cent of the total genetic variation arose from differences between the nominal species $\left(F_{\mathrm{ST}}\right)$ whereas most of the genetic variation (59.2 per cent) was contained within samples. In a second model, we subdivided the total population as described by the phenogram into four subpopulations (samples 1-6, $7-10,11-14$, and $15-20)$. As we would expect, the proportion of gene differentiation between subpopulations increased to 36.8 per cent whereas the genetic variation contained within samples remained at 59.2 per cent. These results suggest that samples included in clusters in the phenogram (Fig. 2) are homogeneous and differentiated from other clusters.

Finally, as measures of gene flow between samples, we estimated $\mathrm{Nm}$ from Wright's $F$-statistics (Table 3) calculated for the entire sample, and the number of migrant individuals per generation by Slatkin's (1985) and Lewontin's (1974) methods. Our Slatkin estimate was based only on samples through the zone of transition between the two nominal species. In this zone, we estimate that in excess of 100 individuals per generation migrate between populations. As an $\mathrm{Nm}$ greater than 0.5 is generally considered sufficient to counteract genetic divergence due to genetic drift (Wright, 1931), there is no apparent impediment to gene flow between species through the zone of transition. Other estimators of $\mathrm{Nm}$ (Lewontin, 1974) based on all 20 samples suggest estimates ranging from 0.632-1.088. Again, even when all 20 samples are considered, we can find no evidence to suggest any impediment to gene flow.

The use of disequilibrium estimates has been recently promoted as a particularly insightful means to investigate 'disturbing forces' within populations (Weir \& Cockerham, 1989), especially within hybrid zones (Barton \& Hewitt, 1989; Szymura \& Barton, 1991). A
Table 3 Summary of Wright's $F$-statistics in Fundulus

\begin{tabular}{lrccc}
\hline Locus & \multicolumn{1}{c}{$F_{\text {IS }}$} & $F_{\mathrm{IT}}$ & $F_{\mathrm{ST}}$ & $N m$ \\
\hline Ada $-A$ & 0.046 & 0.048 & 0.455 & 0.799 \\
sMdh- $A$ & 0.054 & 0.672 & 0.654 & 0.632 \\
Pgm $-A$ & 0.063 & 0.351 & 0.307 & 1.064 \\
sIcdh- $A$ & -0.016 & 0.382 & 0.391 & 0.889 \\
Est-3 & 0.046 & 0.330 & 0.298 & 1.088 \\
Mean & 0.036 & 0.435 & 0.414 & 0.894 \\
\hline
\end{tabular}

total of 1200 disequilibria was estimated (20 populations $\times 10$ pairwise comparisions $\times 6$ types of disequilibria). Of these, most were not significant. A total of 50 (about 4 per cent) of the 1200 exceeded the critical $\chi^{2}$, which is what one would expect by chance alone. From this we conclude that there are no significant disequilibria. Figure 3 shows the pattern of variation of disequilibria from north to south across the hybrid zone. Visual inspection of the figure clearly indicates higher variation of disequilibrium estimates within the hybrid zone than either north or south of the zone. Of the 50 disequilibrium estimates that were significantly larger than zero, 36 were within the hybrid zone.

Inspection of Fig. 3 indicates that disequilibria are likely to be both highest and lowest within the hybrid zone. To test this hypothesis we have transformed all disequilibrium estimates to their absolute values and used the Kruskal-Wallis nonparametric method (sAS, 1988) for the absolute value of $D$ across the hybrid zone. Using this approach we found highly significant effects of hybrid zone $\left(\chi^{2}\right.$ values, $P<0.001$ for all values: $D_{\mathrm{A}}=98, D_{\mathrm{B}}=103, \Delta_{\mathrm{AB}}=84, D_{\mathrm{AAB}}=80$, $D_{\mathrm{ABB}}=98, \Delta_{\mathrm{AABB}}=92$ ). The results are the same if one uses ANOVA.

\section{Discussion}

For each of the five polymorphic loci examined, we could choose a particular allele that represents the most common in each presumed species and follow its change in frequency through the study area. In Fig. 4 we chose the most common allele for each locus in $F$. majalis and plotted its frequency in successive samples around the Florida peninsula. From this representation, it is clear that the allele frequencies vary clinally throughout the study area. The genotypic clines are wide; only $s M d h-A$ is fixed at both ends. This interpretation is consistent with the results of gene flow analyses which suggest that there is no impediment to gene flow.

The $30 \mathrm{~km}$ bounded by the north and south borders of Flagler County, FL, is of particular interest because 

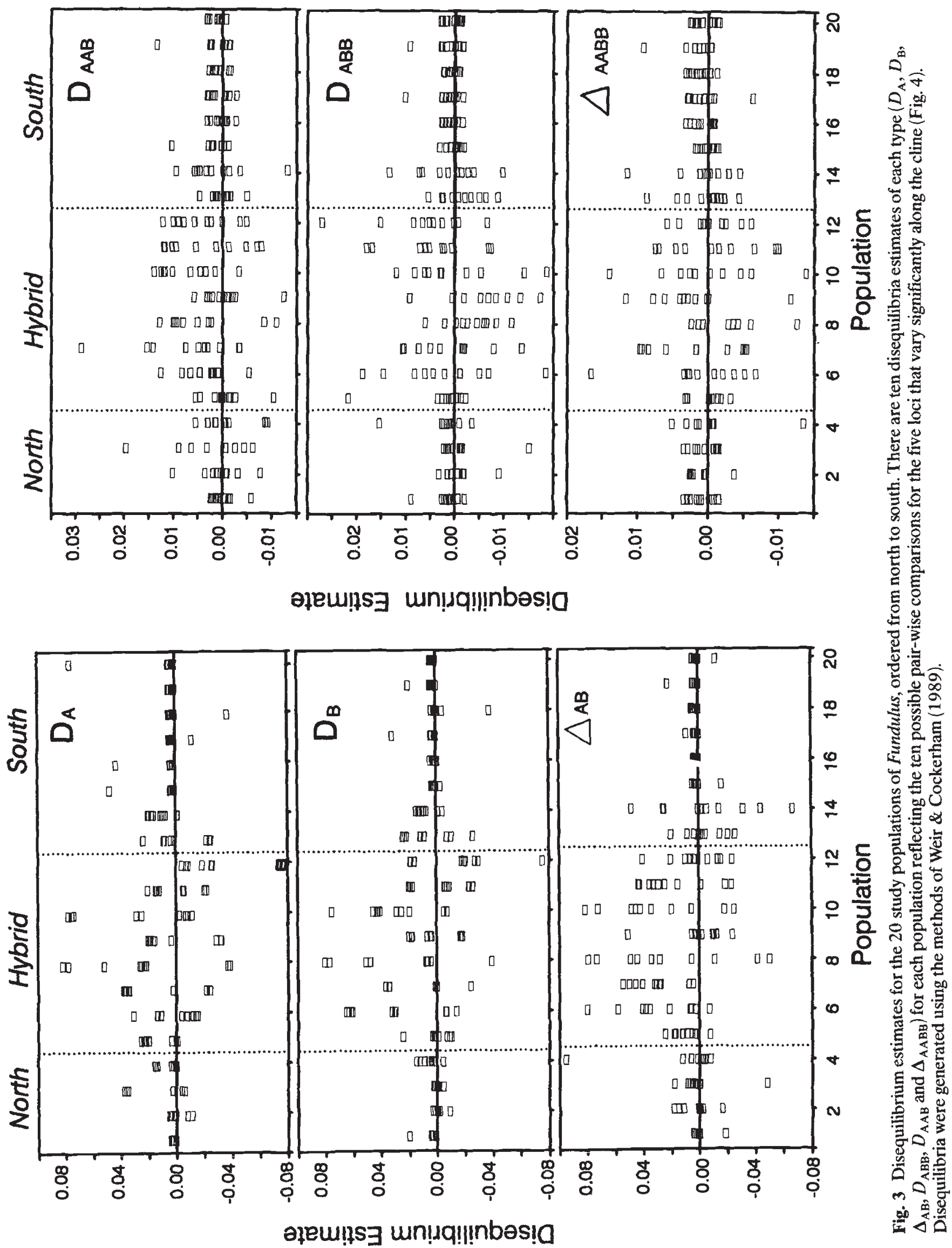


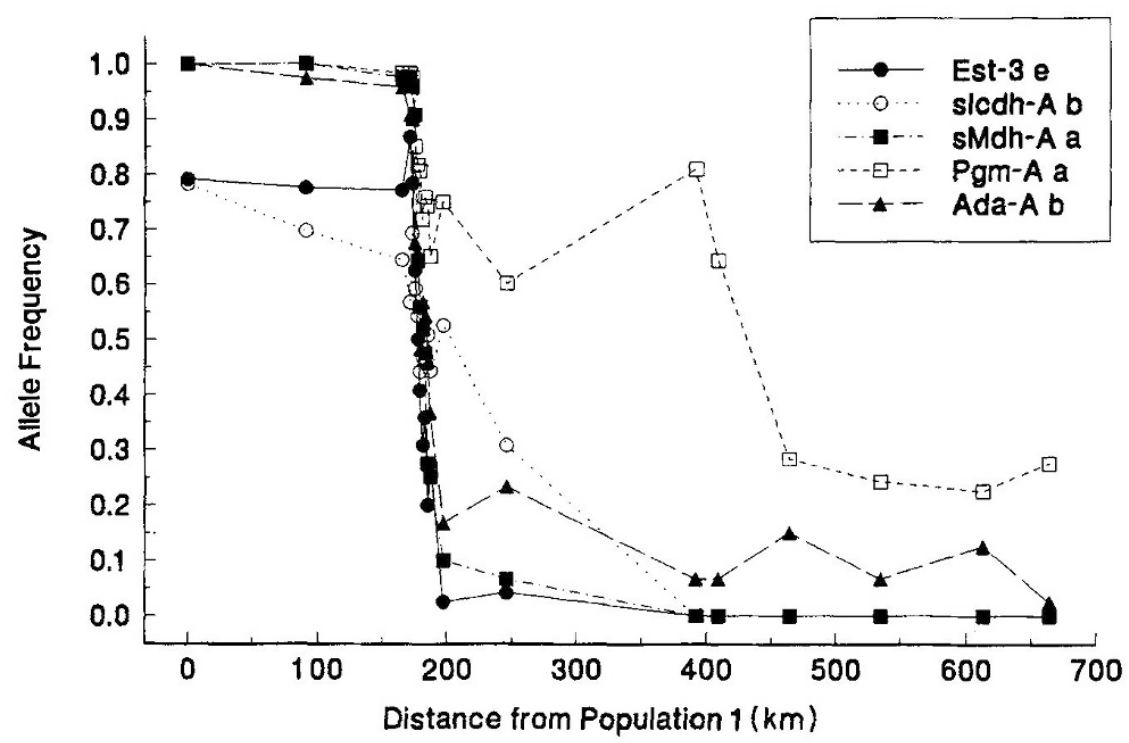

Fig. 4 Graph of allele frequencies for five loci in Fundulus against distance from population 1 (in $\mathrm{km}$ ). it is within this region that we observe the sharpest change in allele frequencies (Fig. 4, samples 4-12). This area represents an ecotone where the Juncus-Spartina marsh characteristic of the northern Atlantic Coast is replaced by mangrove marsh, characteristic of southern peninsular Florida. Thus the maintenance of this zone could be attributed to selection along an environmental gradient (Endler, 1977; Hewitt, 1989). Several studies, including Karl \& Avise (1992), DiMichele et al. (1991) and Brown and Chapman (1991) have shown that allelic isozymes are often adaptive and thus subject to selection.

With only minor exceptions, genotypic frequencies in the Flagler County area and elsewhere throughout the range of sample collections are in Hardy-Weinberg equilibrium. It is possible for genotypic frequencies to be in Hardy-Weinberg equilibrium in a hybrid zone, especially if the hybrid zone is maintained by selection. Barton \& Hewitt (1985) point out that in the bulk of the literature, a hybrid zone is synonymous with a cline. They describe two types of cline: dispersal-independent and tension zones. Dispersal-independent clines show a stable equilibrium of allele frequencies (or other characters) at each locality along the cline. The particular equilibrium point at each location along the cline parallels the change of selection coefficient along an environmental gradient. Tension zones are where the homogenizing effects of dispersal are counteracted by selection against intermediate genotypes (heterozygotes or recombinants). If the cline in the Flagler County area were a tension zone, one would still expect alleles characteristic of each species to occur in sets inherited from the parental forms, in a sense conjoined by inheritance, not linked by physically being on the same chromosome. We tested for this possibility by estimating 'linkage' disequilibria. Most of the significant disequilibria are in the Flagler County area, and the effect is enhanced if one uses the absolute value of the estimates. This analysis was intended to illuminate the pattern of gene flow found with the $F_{\mathrm{ST}}$ analysis. That variance in disequilibria increases suggests dispersal of parental genotypes through the zone. That most disequilibria are not significant suggests that these parental genotypes are freely mixing within their new settings. The absence of significant disequilibria does not support the hypothesis that a tension zone exists in the Flagler County area. Instead, the cline seems to be of the dispersal-independent type. Thus, our data support Relyea's (1983) hypothesis that $F$. majalis and $F$. similis are synonyms.

Historically, there have probably been one or more secondary contacts between two allopatrically differentiated forms, $F$. majalis, a northern Juncus-Spartina marsh form and the nominal $F$. similis, a southern mangrove marsh form (although $F$. similis does occur in Juncus-Spartina marsh in the northern Gulf of Mexico). We speculate that during a previous interglacial epoch when southern Florida became too warm to support a common ancestor to these forms, populations became isolated from each other, one in the northern Gulf of Mexico and the other along the eastern seaboard. Differentiation of these two allopatric populations ensued with the nominal $F$. similis adapting to the environment of the Gulf of Mexico and the nominal $F$. majalis adapting to the environment of the eastern seaboard. Subsequent glaciation and associated climatic cooling displaced the distributions of each southward. Avise (1992) summarizes the effects 
of Pleistocene glaciations on the biota of the southeastern USA). Populations of $F$. similis then dispersed around the southern tip of the Florida peninsula and up the Florida east coast, and eventually recontacted the more northern Atlantic form, the nominal $F$. majalis, somewhere on the east coast of Florida. Evidence of that contact is seen now in the Flagler County area. Apparently the two populations were incompletely differentiated and hybridization between the two previously allopatric forms occurred. The selection pressures that resulted in the original differentiation apparently persist today as evidenced by the clinal variation in allele frequencies through the contact zone (Fig. 4). Clinal variation in allele frequencies in estuarine cyprinodonts may be quite common. Within the distribution of $F$. heteroclitus, Powers et al. (1986) found sharp clines in gene frequency, a complete shift from a northern allele to a southern allele within one degree of latitude. This is not an isolated case of secondary contact through eastern peninsular Florida. Similar cases have been documented for: Fundulus heteroclitus-F. grandis (Duggins et al., 1989), Menidia menidia and $M$. peninsulae (Duggins et al., 1986), Syngnathus (Herald, 1965), Brevoortia (Dahlberg, 1970), Chasmodes (Springer, 1959), Spheroides (Shipp \& Yerger, 1969) and the stone crab, Menippe (Bert \& Harrison, 1988).

The case of the stone crab also parallels this case in terms of morphological characteristics. In each case, coloration and pattern change through the contact zone. In our case, females of the nominal $F$. majalis have vertical bars as juveniles and as the fish matures the bars become longitudinal. Juvenile and adult $F$. similis females have vertical bars. Females in Flagler County display variable phenotypes. Some have close to a typical 'majalis' or 'similis' pattern, others have vertical bars as juveniles, and a series of dots, dashes and irregular markings as adults (Relyea, 1983). Although we collected mostly juvenile individuals, in the few female adults sampled we found no obvious correlation between pigmentation pattern and genotype. Our sample was too small to allow statistical tests.

In conclusion, the clinal pattern of genotypic frequencies, width of the genotypic clines, $\mathrm{Nm}$ values and the absence of significant disequilibria indicate that there are no barriers to gene flow throughout the range of this species complex. The hybrid zone between Fundulus majalis and $F$. similis in the Flagler County area is in an area of rapid ecological transition which results in rapid shifts in genotypes and morphology. The dispersal-independent cline of this hybrid zone is probably maintained by selection against the morphological and physiological characters associated with the different habitats. This hybrid zone could perhaps be best termed an ecological hybrid zone.

\section{Acknowledgements}

The authors acknowledge the financial support for this project from the University of South Carolina and Cameron University, Lawton, Oklahoma. Wesleyan College, Macon, Georgia provided some travel funds. D. Buth and S. Guttman critically commented on early drafts of the manuscript. We also thank the Office of Research in Science and Technology (ORST) at the University of Arkansas at Little Rock for providing laboratory facilities and computer time.

\section{References}

AVISE, J. C. 1992. Molecular population structure and the biogeographic history of a regional fauna: a case history with lessons for conservation biology. Oikos, 63, 62-76.

BARTON, N. H. AND HEWITT G. M. 1985. Analysis of hybrid zones. Ann. Rev. Ecol. Syst., 16, 113-148.

BARTON, N. H. AND HEWITT, G. M. 1989. Adaptation, speciation and hybrid zones. Nature, 341, 497-503.

BERT, T. M. AND HARRISON, R. G. 1988. Hybridization in Western Atlantic stone crabs (genus Menippe): evolutionary history and ecological context influence species interactions. Evolution, 42, 528-544.

BREWER, G. J. 1970. An Introduction to Isozyme Techniques. Academic Press, New York.

BROWN, J. L. 1957. A key to the species and subspecies of the cyprinodont genus Fundulus in the United States and Canada, east of the continental divide. J. Wash. Acad. Sci., 47, 69-77.

BROWN, B. L. AND CHAPMAN, R. W. 1991. Gene flow and mitochondrial DNA variation in the killifish, Fundulus heteroclitus. Evolution, 45, 1147-1161.

BUTH, D. G. AND BERG, w. 3. 1979. Glucose dehydrogenase expression in some marine teleost fishes. Isozyme Bull., 12,71 .

CRABTREE, C. B. AND BUTH, D. G. 1981. Gene duplication and diploidization in tetraploid catastomid fishes Catastomus fumeiventris and C. santaanae. Copeia, 1981, 705-708.

DAHLBERG, M. D. 1970. Atlantic and Gulf of Mexico Menhadens, genus Brevoortia (Pisces: Clupeidae). Bull. Fl. State Mus., 15, 91-162.

DIMICHELE, L., PAYNTER, K. T. AND POWERS, D. A. 1991. Evidence of lactate dehydrogenase-B allozyme effects in the teleost, Fundulus heteroclitus. Science, 253, 898-900.

DUGGINS, C. F., KARLIN, A. A. AND RELYEA, K. G. 1986. Systematics of the Key Silverside, Menidia conchorum, with comments on other Menidia species (pisces: Atherinidae). Tulane Studies in Zoology and Botany, 25, 133-150.

DUGGins, C. F., KARLIN, A. A. AND RELYEA, K. G. 1989. Biochemical systematics in southeastern populations of Fundulus heteroclitus and Fundulus grandis. Northeast Gulf Science, 10, 95-102.

ENDLER, J. A. 1977. Geographic Variation, Speciation and Clines. Princeton University Press, Princeton, NJ. 
FISHER, S. E, SHAKLEE, J. B., FERRIS, S. D. AND WHITT, G. S. 1980. Evolution of five multilocus isozyme systems in the chordates. Genetica, 52/53, 73-85.

HARris, R. J. 1985. A Primer of Multivariate Statistics. Harcort Brace Jovanovich, San Diego, CA.

HERALD, E. S. 1965. Studies on the Atlantic American pipefishes with descriptions of new species. Proc. Calif. Acad. Sci., 32, 363-375.

HEwITT, G. M. 1989. The subdivision of species by hybrid zones. In: Otte, D. and Endler, J. A. (eds) Speciation and its Consequences, pp. 85-110. Sinauer, Sunderland, MA.

JACKSON, C. F. 1953. Northward occurrence of southern fishes (Fundulus, Mugil, Pomatomus) in coastal waters of New Hampshire. Copeia, 1953, 192.

KARL, S. A. AND AVISE. J. C. 1992. Balancing selection at allozyme loci in oysters: implications from nuclear RFLPS. Science, 256, 100-102.

LEWONTin, R. C. 1974. The Genetic Basis of Evolutionary Change. Columbia University Press, New York.

MILLER, R. R. 1955. An annotated list of the American cyprinodontid fishes of the genus Fundulus, with description of Fundulus persimilis from Yucatan. Occ. Pap. Mus. Zool. Univ. Mich., 568, 1-25.

NEI, M. 1978. Estimation of average heterozygosity and genetic distance from a small number of individuals. Genetics, 89, 583-590.

POWERS, D. A., ROPSON, I., BROWN, D. C., BENEDEN, R. V., CASHON, R., GONZALEZ-VILLASENOR, L. I. AND DIMICHELE, J. A. 1986. Genetic variation in Fundulus heteroclitus: geographic distribution. Am. Zool., 26, 131-144.

RELYEA, K. R. 1983. Systematic study of two species complexes of the genus Fundulus. Bull. Florida State Mus., 29, 1-64.

SAS INSTITUTE. 1989. SAS/STAT User's Guide, Version 6, 4th edn. SAS Institute, Cary, NC.
SELANDER, R. K., SMITH, M. H., YANG, S. Y., JOHNSON, W. E. AND GENTRY, J. B. 1971. Biochemical polymorphism and systematics in the genus Peromyscus. I. Variation in the old-field mouse Peromyscus polionotus. Stud. Genetics VI. Univ. Texas Publ, 7103, 49-90.

SHIPP, R. L. AND YERGER, R. w. 1969. Status, characters and distribution of the northern and southern puffers of the genus Sphoeroides. Copeia, 1969, 425-433.

SLATKIN, M. 1985. Gene flow in natural populations. Ann. Rev. Ecol. Syst., 16, 393-430.

SPRINGER, V. C. 1959. Blenniid fishes of the genus Chasmodes. Texas J. Sci., 11, 321-334.

SWOFFORD, D. L. AND SELANDER, R. B. 1981. BIOSYS-1: A Fortran program for the comprehensive analysis of electrophoretic data in population genetics and systematics. J. Hered., 72, 281-283.

SZYMURA, J. M. AND BARTON, N. H. 1991. The genetic structure of the hybrid zone between the fire-bellied toads Bombina bombina and $B$. variegata:comparisions between transects and between loci. Evolution, 45, 237-261.

WAHLUND, s. 1928. The combination of populations and the appearance of correlation examined from the standpoint of the study of heredity. Hereditas, 11, 65-106.

WEIR, B. S. 1990. Genetic Data Analysis. Sinauer, Sunderland, MA.

WEIR, B. S. AND COCKERHAM, C. C. 1989. Complete characterization of disequilibrium at two loci. In: Feldman, M. W. (ed.) Mathematical Evolutionary Theory, pp. 86-110. Princeton University Press, Princeton, NJ.

WRIGHT, s. 1931. Evolution in Mendelian populations. Genetics, 6, 111-178.

WRIGHT, s. 1978. Evolution and the Genetics of Populations, vol. 4, Variability Within and Among Natural Populations. University of Chicago Press, Chicago. 